\title{
Modelling the Effects of Hypertension on Ventricle Cells of Human Heart
}

\author{
Hamsa N Naser, Yinhua Zhang, Henggui Zhang \\ The University of Manchester, Manchester, UK
}

\begin{abstract}
Systemic hypertension (HP) is one of the key risk factors for evolving cardiac hypertrophy and heart failure (HF). Previous studies have shown that cardiac hypertrophy is associated with altered excitation-contraction (E-C) coupling and enhanced myocardial contraction, whilst HF is associated with diminished contractility.

This study aimed to investigate the functional impact of hypertension on the cardiac mechanical dynamics and the contracting mechanisms of the left ventricular myocytes of human heart. An electromechanical model of human ventricular cell model developed by Adeniran et al. has been modified by incorporating available experimental data from rat ventricular cells under Sham (control) and HP conditions. Hypertrophy was modeled by incorporating experimental data of changes in $I_{N a}, I_{t o}$, $\mathrm{I}_{\mathrm{NaCa}}$, cell size and myofilament responses to $\mathrm{Ca}^{2+}$ in the cardiac cell.

Simulations showed that HP produced:1) prolongation of the action potential duration at $90 \%$ repolarization $\left(A P D_{90}\right)$ by approximately $\left.4.7 \% ; 2\right)$ an increase of the intracellular calcium concentration $\left(\left[\mathrm{Ca}^{2+}\right]_{i}\right)$ by $36 \%$; 3) no marked change in the sarcomere length or the contractile force. Simulation results were consistent with experimental data, validating the HP model development and indicated the pro-arrhythmic effects of HP.
\end{abstract}

\section{Introduction}

Systemic hypertension (high blood pressure, HP) represents one of the vital risk factors for evolving cardiac hypertrophy and heart failure (HF). The impact of hypertensive heart disease is emphasised by its high dominance and complications [1]. It has been found that in the developed countries such the USA (United State of America), a $30 \%$ of adult have hypertension and $60 \%$ of these develop cardiac hypertrophy, causing a substantial disease problem [1]. Numerous studies [2-6] have denoted that cardiac hypertrophy is linked to changes in the excitation-contraction (E-C) coupling and enhanced myocardial contraction, while HF is linked to diminished contractility.
These findings indicate that a direct relation between the hypertension onset and the $\mathrm{Ca}^{2+}$ signaling upregulation that probably lead to hypertrophy and HF when prolonged exposure to hypertension Systemic blood [2].

However, the causative cellular and molecular mechanisms linking hypertension to HF are still unclear. It has been found that HP induces a left ventricular (LV) hypertrophy, which causes remodeling of several ion channels as well as prolongs the ventricles depolarization [7]. Such remodeling has been reported in the ventricular myocytes of some mammal species such as rats where hypertrophy induces changes in the density of several currents including, $I_{N a}, I_{t o}$ and $I_{N a C a}$, in addition to changes in the cell size and the calcium sensitivity of Ltype $\mathrm{Ca}^{2+}$ which is myofilament responses to $\mathrm{Ca}^{2+}$ in the cardiac cell [7]. Therefore, to simulate hypotrophy, all these changes have been employed in the electromechanical model for human ventricular myocytes in the current paper.

In this study, electromechanical model of the O'HaraRudy (ORd) human ventricle model, previously developed by Adeniran et al. [8], were utilised to investigate the hypertension effects on the cardiac mechanical dynamics, the mechanisms of contracting and force-generating of the human ventricular cells. The ORd electromechanical model modification based on experimental data from the rat LV experimental data under Sham (control) and hypertension conditions from Jin et al. work [3].

\section{Methods}

\subsection{Model development}

In order to obtain an electromechanics model that can describe the human heart contraction, the ORd electrophysiological model of the human ventricular myocytes [9] was coupled to the myofilament model [10]. The resulted electromechanics model has been modified by integrating available experimental findings from rat ventricular myocytes under Sham and hypertension conditions [3].

Firstly, changes in the myofilament responses to $\mathrm{Ca}^{2+}[3]$ have been incorporated in Sham and HP models 
(not shown in this paper). Secondly, followed the work of Kharche et al., 2005 [7] and to simulate LV hypertrophy, changes include sodium channel current, $I_{N a}$, up- regulated (conductance increased by $8 \%$ ), the outward transient potassium channel current, $\mathrm{I}_{\text {to }}$, down-regulated (conductance decreased by $35 \%$ ), and sodium-calcium exchanger current up-regulated (the $I_{\mathrm{NaCa}}$ scaling factor increased by $5 \%$ ), with a $30 \%$ increase in the cell size and cell capacitance [7], all of which have been implemented in the hypertension model.
Simulation results show that HP induce: 1) a reduction in the myofilament $\mathrm{Ca}^{2+}$ sensitivity by $34 \%[11,12], 2$ ) prolongation of the $\mathrm{APD}_{90}$ by approximately $4.7 \%$, as shown in Figure 1A, 3) no obvious change in the sarcomere length (SL), as the initial SL was $2.2 \mu \mathrm{m}$ and the smallest contracted SL was $2.14 \mu \mathrm{m}$, however, there was a mark decrease by $13.7 \%$, in the time course of the sarcomere length in HP condition than in Sham condition, as illustrated in Figure 1B,
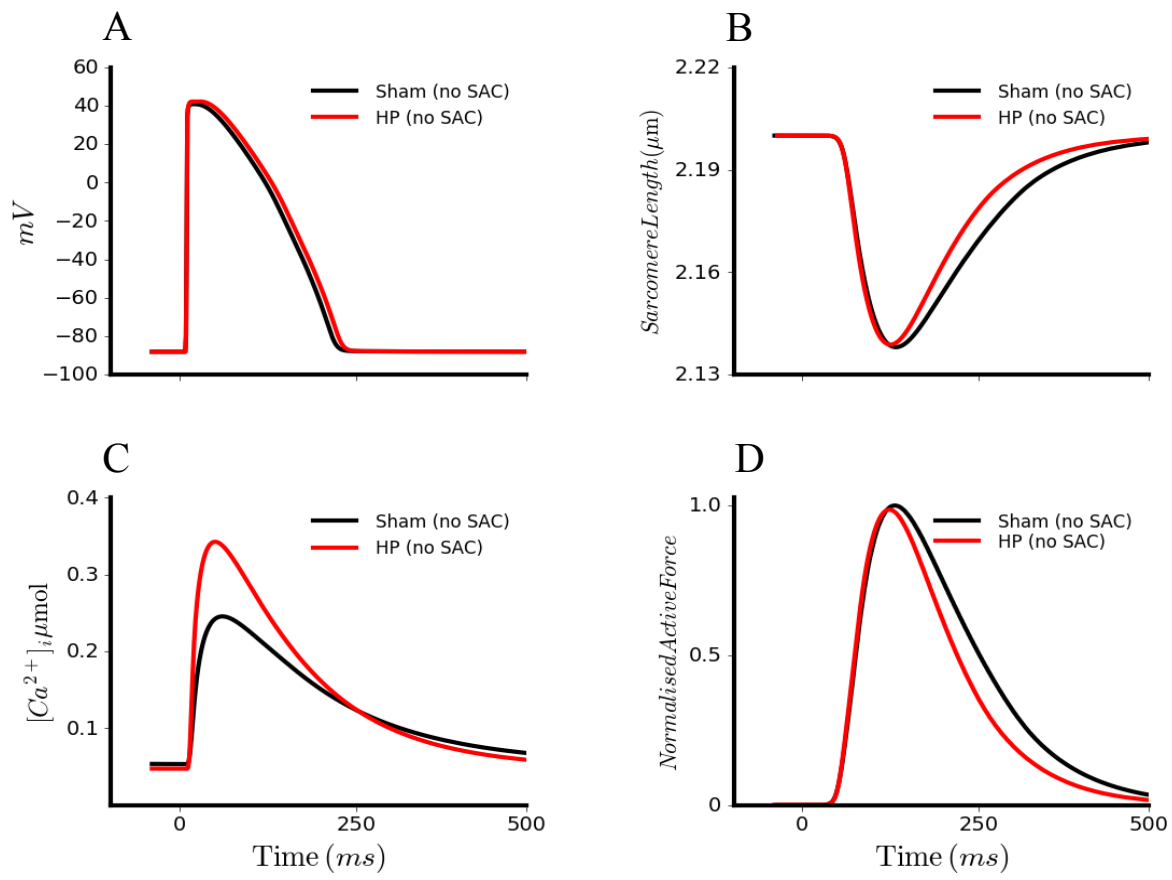

Figure 1: Single cell electromechanical effects of the HP without $I_{s a c}$. Simulated (A) normalised active force and $\left(\left[\mathrm{Ca}^{2+}\right]_{\mathrm{i}}\right)$ relationship, (B) AP, (C) calcium transient $\left(\left[\mathrm{Ca}^{2+}\right]_{\mathrm{i}}\right),(\mathrm{D})$ sarcomere length $(\mathrm{SL})$ and (E) active force. ( $\mathrm{F}$ and $\mathrm{G}$ ) the experimental time course of SL and $\left[\mathrm{Ca}^{2+}\right]_{i}$, respectively, which elicited by an action potential in rat ventricular myocytes from [3].

\section{Results}

\subsection{Single cell simulations}

Simulated APs were obtained with a $1-\mathrm{Hz}$ stimulation frequency and calculated after at least 5 minutes of continuous simulation to make sure that a steady-state solution was reached. The functional impact of the hypertension on the action potential duration, cardiac sarcomere length, the intracellular calcium concentration $\left(\left[\mathrm{Ca}^{2+}\right]_{\mathrm{i}}\right)$, the contractile force and the myofilament responses to $\mathrm{Ca}^{2+}$ (calcium sensitivity of L-type $\mathrm{Ca}^{2+}$ ), were investigated in this paper without considering the effects of the stretch-activated channels (SACs).
4) an elevation in the peak of the intracellular calcium concentration $\left(\left[\mathrm{Ca}^{2+}\right]_{\mathrm{i}}\right)$ by $36 \%$ with a faster relaxation and time constant of $\left(\left[\mathrm{Ca}^{2+}\right]_{\mathrm{i}}\right.$ decay (tau), as in Figure $\left.1 \mathrm{C}, 5\right)$ no significant alteration in the contractile force peak while there was a $12.8 \%$ decrease in the time course of the normalised active force (which is the contractile force normalised to its maximum value) in HP condition than Sham condition, as in Figure 1D.

Indeed, the results of unaltered the amplitude of sarcomere length and the contractile force suggest that there is unchanged in the cardiac myocytes contraction of the LV under HP condition [3].

All these simulations were in a good agreement with the experimental data from [3] and thus validated the 
electromechanical models under both Sham and HP conditions. A summary of these results is shown in Figure 2 , which shows a comparison between the simulation results presented in the pink columns while the experimental data are the blue squares.

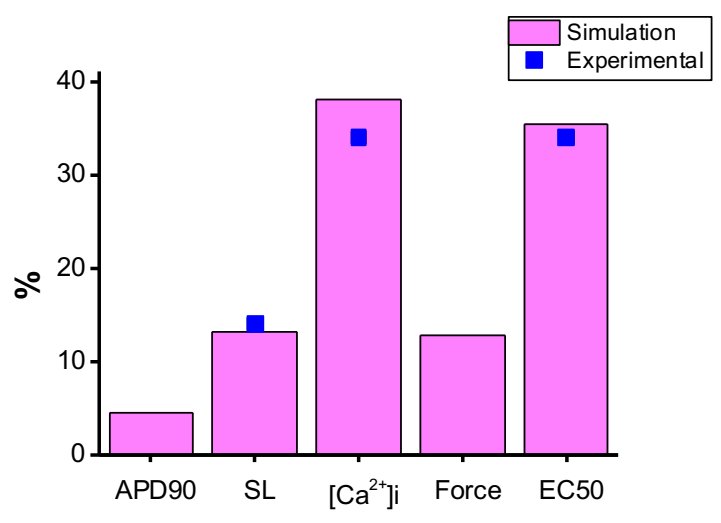

Figure 2: Bar plots showing the effects of hypertension on human left ventricular myocytes. It illustrates the percentages of simulation results (pink columns) and experimental data (blue squares) from Jin et al. [3].

Figure 3A shows the steady state action potential duration (APD) rate-dependence for the $\mathrm{APD}_{90}$ of the human LV electromechanical models under Sham and HP conditions. The APD restitution curve shows the APD recovery as a function of basic cycle length (BCL).

The $\mathrm{APD}_{90}$ restitution curves, under both Sham and HP conditions, demonstrate that at BCLs below $600 \mathrm{~ms}$, the model demonstrates a great rate dependency and above 600 ms it becomes more stable.

Moreover, simulations show that the prolongation of the LV cardiac cells under HP condition, resulted in a leftward shift of the rate dependency at all BCLs examined and increased the maximal slope, producing an $A P D_{90}$ restitution curves less flattened in hypertension than Sham conditions, as shown in Figure 3B.

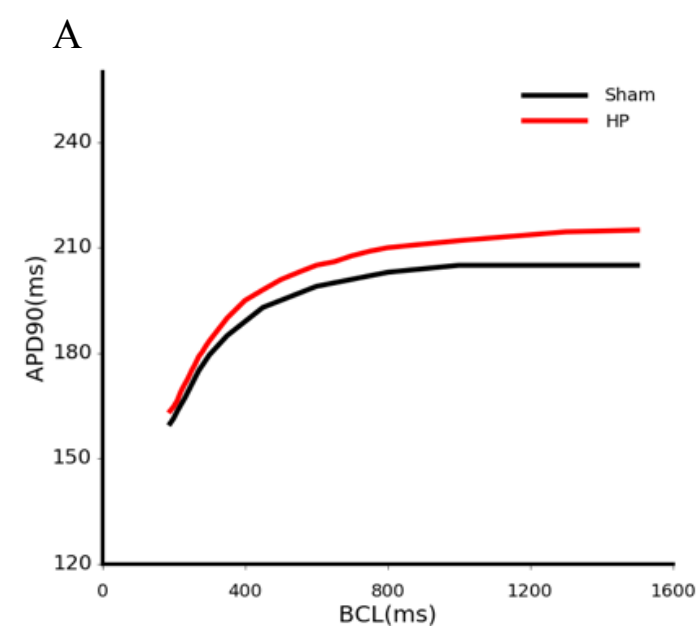

\section{$\mathrm{B}$}

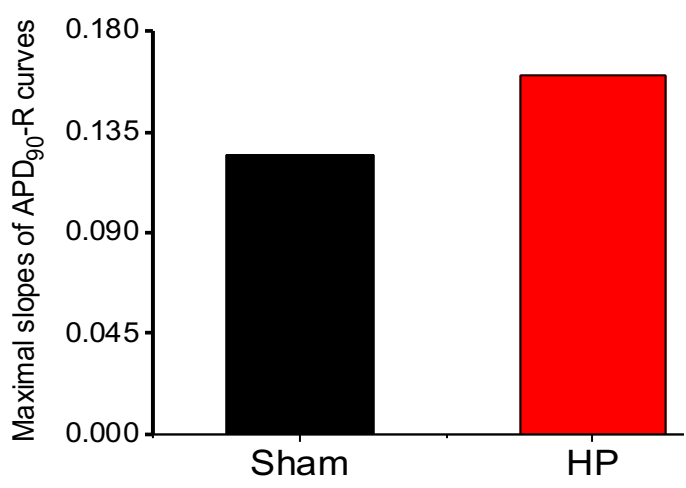

Figure 3: Steady state APD rate dependence curves of Sham (black) and HP (red) electromechanical models, demonstrating the cycle length dependency of the $\mathrm{APD}_{90}$ for the LV of the human heart in (A). While the maximal slopes of the APD restitution curves of both Sham and HP are shown in (B).

\section{Discussion and conclusion}

The ORd electromechanical model [8] of human ventricular cells was used in this paper to investigate the functional impact of hypertension on the contractile function of the LV human heart without taking into consideration the effects of SACs.

This model has been modified according to available experimental data from rat ventricular cells [3], under Sham and HP conditions. The LV hypotrophy that induced by HP was modeled following the work of Kharche et al. [7] and then implemented in the HP electromechanical model.

Simulations showed that HP condition prolonged the 
$\mathrm{APD}_{90}$ and increased the $\left[\mathrm{Ca}^{2+}\right]_{\mathrm{i}}$ peak with no significant change to the sarcomere length or the normalized active force, indicating that HP condition might not modulate the contraction of the LV cardiac myocytes.

These results of human LV match qualitatively to those empirically observed from rat ventricular cell, validating the models development [3]. These results indicate the proarrhythmic effects of HP condition, providing mechanistic insights into a better understanding to the increase risk of ventricular fibrillation in hypertensive patients.

The electromechanical models in this study can be further used in the investigations of the effects of hypertension combined the SACs effects at single cell, two-or three dimensional models of the intact human ventricular myocytes, then in the study of the whole heart contractile function.

\section{Acknowledgment}

This project was supported by The Higher Committee for Education Development in Iraq (HCED).

\section{References}

[1] Yildirir A. Hypertension and Arrhythmia: Blood Pressure Control and Beyond. Europace 2002; 4: 175-182.

[2] Chen-Izu Y, Chen L, Bányász T, et al. HypertensionInduced Remodeling of Cardiac ExcitationContraction Coupling in Ventricular Myocytes Occurs Prior to Hypertrophy Development. Am J Physiol-Heart Circ Physiol 2007; 293: H3301$\mathrm{H} 3310$.

[3] Jin CZ, Jang JH, Kim HJ, et al. Myofilament $\mathrm{Ca}^{2+}$ Desensitization Mediates Positive Lusitropic Effect of Neuronal Nitric Oxide Synthase in Left Ventricular Myocytes from Murine Hypertensive Heart. J Mol Cell Cardiol 2013; 60: 107-115.

[4] Gómez AM, Schwaller B, Porzig H, et al. Increased Exchange Current but Normal $\mathrm{Ca}^{2+}$ Transport via $\mathrm{Na}^{+}-\mathrm{Ca}^{2+}$ Exchange During Cardiac Hypertrophy after Myocardial Infarction. Circ Res 2002; 91: 323-330.

[5] Li Q, Keung EC. Effects of Myocardial Hypertrophy on Transient Outward Current. Am J Physiol-Heart
Circ Physiol 1994; 266: H1738-H1745.

[6] Yokoshiki H, Kohya T, Tomita F, et al. Restoration of Action Potential Duration and Transient Outward Current by Regression of Left Ventricular Hypertrophy. J Mol Cell Cardiol 1997; 29: 13311339.

[7] Kharche S., Zhang H., Clayton RC., and Holden A.V.(1998). Hypertrophy in Rat Virtual Left Ventricular Cells and Tissue. In Pritchard, D., \& Reeve, J. (Eds) 1998. Euro-Par '98 parallel processing: 4th International Euro-Par Conference Southampton, UK, September 1-4, 1998; 1470 proceedings.

[8] Adeniran I, Hancox JC, Zhang H. Effect of Cardiac Ventricular Mechanical Contraction on The Characteristics of The ECG: A Simulation Study. J Biomed Sci Eng 2013; 06: 47-60.

[9] O'Hara T, Virág L, Varró A, et al. Simulation of the Undiseased Human Cardiac Ventricular Action Potential: Model Formulation and Experimental Validation. PLoS Comput Biol 2011; 7: e1002061.

[10] Rice JJ, Wang F, Bers DM, et al. Approximate Model of Cooperative Activation and Crossbridge Cycling in Cardiac Muscle Using Ordinary Differential Equations. Biophys $J$ 2008; 95: 23682390.

[11] Chung J-H, Biesiadecki BJ, Ziolo MT, et al. Myofilament Calcium Sensitivity: Role in Regulation of In vivo Cardiac Contraction and Relaxation. Front Physiol; 7. Epub ahead of print 16 December 2016.2 DOI: 10.3389/fphys.2016.00562.

[12] Jin CL, Yin MZ, Paeng JC, et al. Neuronal Nitric Oxide Synthase Modulation of Intracellular $\mathrm{Ca}^{2+}$ Handling Overrides Fatty Acid Potentiation of Cardiac Inotropy in Hypertensive Rats. Pflüg Arch - Eur J Physiol 2017; 469: 1359-1371.

Address for correspondence.

Hamsa Naser

3.06. Schuster Laboratory, University of Manchester, Manchester, UK, M13 9PL, hamsa.naser@postgrad.manchester.ac.uk 